\title{
A C*-Algebra Approach to the Schwinger Model
}

\author{
A. L. Carey ${ }^{1}$ and C. A. Hurst ${ }^{2}$ \\ 1 Department of Pure Mathematics, The University of Adelaide, 5001 Adelaide, South Australia \\ 2 Department of Mathematical Physics, The University of Adelaide, 5001 Adelaide, South Australia
}

\begin{abstract}
If cutoffs are introduced then existing results in the literature show that the Schwinger model is dynamically equivalent to a boson model with quadratic Hamiltonian. However, the process of quantising the Schwinger model destroys local gauge invariance. Gauge invariance is restored by the addition of a counterterm, which may be seen as a finite renormalisation, whereupon the Schwinger model becomes dynamically equivalent to a linear boson gauge theory. This linear model is exactly soluble. We find that different treatments of the supplementary (i.e. Lorentz) condition lead to boson models with rather different properties. We choose one model and construct, from the gauge invariant subalgebra, a class of inequivalent charge sectors. We construct sectors which coincide with those found by Lowenstein and Swieca for the Schwinger model. A reconstruction of the Hilbert space on which the Schwinger model exists is described and fermion operators on this space are defined.
\end{abstract}

\section{Introduction}

In a series of previous papers [1-6] we have developed a definite metric quantisation procedure for linear boson field theories which contain massless particles. In the case of the free electromagnetic field this rigorises Fermi's original quantisation procedure [7] and among other things provides a natural framework for a discussion of gauge transformations [3], [4]. It has an advantage over the indefinite metric approach in that standard $C^{*}$ algebra and Hilbert space methods may be applied.

To see how these ideas might work in a fully interacting theory we decided to attempt an analysis of the Schwinger model [8] [(QED) $)_{2}$ with massless Fermions] and (QED) $)_{2}$ itself. In this paper we discuss only the Schwinger model from our viewpoint. There is a fortunate accident which occurs as a consequence of the masslessness of the Fermions in the Schwinger model. Namely the model may be "bosonised" in the sense that, as far as the observables of the model are concerned, the dynamics can be expressed solely in terms of currents without reference to the fermion fields themselves. This possibility does not seem to be 
open in (QED) 2 and there more subtle methods seem to be required. Now the Schwinger model has received quite a lot of attention recently (we cite for example [9-11] and references listed therein). What we are going to say is new in the sense that we will give a detailed account of the structure of the model as a local gauge theory and show how the ideas of Doplicher, Haag and Roberts [12] may be applied so as to reconstruct the charge sectors of the model from the gauge invariant algebra.

In our approach the usual difficulties of zero mass field theories [13], [14] manifest themselves. Our philosophy is however, that these difficulties occur because one is trying to impose physical constraints on unobservable quantities. From this viewpoint the field algebra, which contains unobservable fields (e.g. in the case of QED, the electromagnetic potential), should be constructed to satisfy a version of the Haag-Kastler axioms (i.e. to have a local structure) as an abstract algebra, while its representations and the field equations should be regarded as adjuncts to constructing the observable algebra and its dynamics.

We remark that our approach to $(\mathrm{QED})_{4}$ (as sketched in [5]) would be to emphasise the local gauge invariance of the theory (in the sense of exploiting harmonic analysis on the gauge group). In this respect we differ from Fröhlich [15].

The paper is organised as follows. In Sect. 1 we discuss the dynamics of the Schwinger model. We not that the interaction part of the "bare" Hamiltonian for the Schwinger model is not locally gauge invariant. The requirements of gauge and Poincaré invariance eventually lead us to an equivalent Boson model. In fact we consider two versions of the Lagrangian for the Schwinger model. The first has a gauge fixing term of the form $\left(\partial^{\mu} A_{\mu}\right)^{2}$ appropriate to the Fermi method for quantising the electromagnetic field [7]. The second introduces an auxiliary field and has a gauge fixing term of the form $\xi(x) \partial^{\mu} A_{\mu}(x)$. This second version turns out to be "smoother" than the first in that the former Lagrangian leads to a Boson model in which the dynamics cannot be implemented in any obvious irreducible representation of the field algebra. Interestingly enough both Lagrangians lead to the same algebra of observables and at this level it is the requirement of time translation invariance of the appropriate states which distinguishes them.

We note that Lundberg [10] has also "bosonised" the Schwinger model, however he works in radiation gauge and, as will become clear in our treatment, it is precisely the properties of the Schwinger model as a local gauge theory that make possible the reconstruction of the charge sectors by an algebraic approach. These sectors do not seem to be "accessible" from a radiation gauge representation.

Most of the results of Sect. 2 are in fact well known. Consequently we have only sketched our argument briefly. In Sect. 3 we discuss the Boson models which arise from each Lagrangian. These models are themselves local gauge theories with quadratic Hamiltonians. Manuceau's variant [16] of Segal's Weyl algebra formalism [17] is used to construct field algebras. We then concentrate on the model which leads to an implementable dynamics while the treatment of the other is only sketched. We follow our discussion [6] of the same model in 4 dimensions. Most of the results of [6] carry over without change although there are a number of important differences in our viewpoint in the 2-dimensional case.

In the final section we reconstruct the charge sectors of the Schwinger model from automorphisms of the gauge invariant subalgebra, say $a$, of the field algebra 
for the boson model. We find that, with an appropriate choice of gauge, we can relate our results to those of Lowenstein and Swieca [18]. This gauge freedom in our approach is a consequence of the fact that $a$ has a non-trivial centre. We necessarily start from a faithful (but not irreducible) cyclic representation of $a$ in order to reconstruct charge sectors. It is possible that this departure from the approach of [12] is typical of abelian local gauge theories. We conclude with a sketch of the reconstruction of the Fermion fields themselves. We are guided here by the results of [18]. Unfortunately our construction is ad hoc, as we have not found a method of characterising the Fermion fields in an algebraic way.

There is one aspect of our work we would like to emphasise here. In all previous rigorous approaches to field theories containing massless particles at least one of three expedients is adopted:

(a) eliminate massless fields by giving them masses

(b) work in Coulomb gauge

(c) use an indefinite metric.

Now (a) and (b) automatically destroy the feature of greatest importance, namely local gauge invariance, while (c) leads to "gauge automorphism" implemented by unbounded operators and hence serious technical difficulties. In this paper we succeed in avoiding all these expedients.

\section{The Schwinger Model}

In this section we discuss various aspects of the model which will provide an appropriate starting point for the rigorous discussion of the remaining sections. Although most of the statements made in this section are well known and, if a space cutoff is introduced, are rigorous, there seem to be some difficulties involved in removing the cutoff. We will see that there is a well defined path from an appropriate linear boson model to a theory of interacting photon and fermion fields but that the converse argument still contains gaps.

As mentioned in the introduction there are two formulations for the Schwinger model which lead to the same theory of observables and each has its advantages. The first involves the Fermi method for quantising the electromagnetic field which follows closely our treatment [1] of the free electromagnetic field in $3+1$ dimensions while the second is the so-called Landau gauge method [9]. The Fermi method in this context gives a linear boson model in which the existence of a pure ground state or vacuum for the Hamiltonian is problematical. We suspect that this is a feature of 2 dimensions only. The Landau gauge method does not suffer from this difficulty.

The classical Lagrangian densities for the two models are

$$
\begin{aligned}
& \mathscr{L}_{\text {Fermi }}=-\frac{1}{2} A_{\mu, v}\left(A^{\mu, v}-A^{v, \mu}\right)-\frac{1}{2}\left(\partial_{\mu} A^{\mu}\right)^{2}+i \psi^{+} \gamma^{\mu}\left(\partial_{\mu}-i e A_{\mu}\right) \psi \\
& \mathscr{L}_{\text {Landau }}=-\frac{1}{2} A_{\mu, v}\left(A^{\mu, v}-A^{v, \mu}\right)+\xi \partial_{\mu} A^{\mu}+i \psi^{+} \gamma^{\mu}\left(\partial_{\mu}-i e A_{\mu}\right) \psi
\end{aligned}
$$

where

$$
\gamma^{0}=\left(\begin{array}{rr}
1 & 0 \\
0 & -1
\end{array}\right), \quad \gamma^{1}=\left(\begin{array}{rr}
0 & 1 \\
-1 & 0
\end{array}\right), \quad \gamma^{5}=\gamma^{0} \gamma^{1}=\left(\begin{array}{ll}
0 & 1 \\
1 & 0
\end{array}\right)
$$


and

$$
\psi^{+}=\bar{\psi} \gamma^{0}
$$

The conjugate momenta are

$$
\begin{array}{lll}
\pi_{0}=-\dot{A}^{0}-\nabla A, & \pi_{1}=\dot{A}+\nabla A^{0}, & \pi=i \bar{\psi}, \\
\pi_{0}=\xi, & \pi_{1}=\dot{A}+\nabla A^{0}, & \pi=i \bar{\psi}
\end{array}
$$

respectively, where $\nabla \equiv \partial_{1}$ and $A \equiv A^{1}$. As the first Eq. of (2.3b) is a Dirac constraint, further discussion requires the use of the Dirac formalism (cf. discussion in [6]), and so for the present the more straightforward case of the Fermi method will be considered.

From (2.1a) and (2.3a) the Hamiltonian is

$$
\begin{aligned}
H= & H_{A}+H_{\psi}+H_{I} \\
= & \int_{-\infty}^{\infty} d x\left[-\frac{1}{2} \pi_{0}^{2}+\frac{1}{2} \pi^{2}-\pi_{1} \nabla A^{0}-\pi_{0} \nabla A\right. \\
& \left.\quad-\pi \gamma^{5} \nabla \psi+i e\left(\pi \psi A^{0}-\pi \gamma^{5} \psi A\right)\right]
\end{aligned}
$$

Now by introducing cutoffs (specifically, putting the system in a box and replacing the spatial continuum by a lattice) (2.4) gives a well-defined operator on the tensor product $\mathscr{F}_{A} \otimes \mathscr{F}_{\psi}$ of the Fock spaces for photons and fermions (see for example Ito [9]). However it is not difficult to see that (2.4) cannot define a sensible dynamics. Included in $H_{I}$ is the current $j^{\mu}$ :

with

$$
H_{I}=\int d x j^{\mu}(x) A_{\mu}(x)
$$

$$
j^{\mu}(x)=-i e: \psi^{+}(x) \gamma^{\mu} \psi(x):
$$

The smeared version of (2.6) (for $f: \mathbb{R} \rightarrow \mathbb{R}^{2}$ smooth of fast decrease)

$$
j(f)=\frac{1}{\sqrt{2 \pi}} \int_{-\infty}^{\infty} d k \int_{-\infty}^{\infty} d k^{\prime} \sum_{\alpha, \beta=1}^{2} a_{\alpha}(k)^{*} u_{\alpha}^{+}(k) \gamma^{\mu} u_{\beta}\left(k^{\prime}\right) a_{\beta}\left(k^{\prime}\right) f_{\mu}\left(k-k^{\prime}\right)
$$

has been often discussed and it is known [19] that if we take the Fock representation for the Fermions then $j(f)$ is self adjoint with commutation relations (in terms of Fourier transforms)

$$
\left[\tilde{j}^{0}(k), \tilde{j}^{1}\left(k^{\prime}\right)\right]=\frac{k}{\pi} \delta\left(k+k^{\prime}\right)
$$

Here $a_{1}(k)$ and $a_{2}(k)$ are the usual fermion and anti-fermion annihilation operators and

$$
u_{1}(k)=\frac{1}{\sqrt{2}}\left(\begin{array}{c}
1 \\
\varepsilon(k)
\end{array}\right), \quad u_{2}(k)=\frac{1}{\sqrt{2}}\left(\begin{array}{c}
1 \\
-\varepsilon(k)
\end{array}\right) .
$$

It is also well known [20] that the commutation relations (2.7) are a consequence of choosing the Fock representation for the fermions, and with different representations, different Schwinger terms are obtained (a good account of this phenomenon 
is contained in [10]). (The current operators are affiliated with the weak closure of the fermion algebra in a quasifree representation). Furthermore, as a consequence of (2.7), the contribution to the equations of motion for the current operators, from the free fermion Hamiltonian $H_{\psi}$, is obtained when $H_{\psi}$ is replaced by a purely boson Hamiltonian according to Kronig's identity [21]. In fact, if a scalar massless boson field $\phi$ is defined by

$$
j^{\mu}(x)=\partial^{\mu} \phi(x)
$$

then (2.4) can be replaced by

$$
\begin{aligned}
H_{\text {boson }}=\int_{-\infty}^{\infty} d x\left[-\frac{1}{2} \pi_{0}^{2}+\frac{1}{2} \pi_{1}^{2}+\frac{1}{2}\left(\pi_{\phi}-g A^{0}\right)^{2}-\pi_{1} \nabla A^{0}-\pi_{0} \nabla A\right. \\
\left.+\frac{1}{2}(\nabla \phi-g A)^{2}-\frac{1}{2} g^{2}\left(\left(A_{0}\right)^{2}+A^{2}\right)\right]
\end{aligned}
$$

where $g^{2}=e^{2} / \pi$. (With cutoffs present this may be proved rigorously cf. [19]). Now (2.10) arises, as a classical Hamiltonian, from the boson field theory with Lagrangian

$$
\begin{aligned}
\mathscr{L}_{\text {Boson }}=\int_{-\infty}^{\infty} d x[ & -\frac{1}{4} F^{\mu v} F_{\mu \nu}-\frac{1}{2}\left(\partial^{\mu} A_{\mu}\right)^{2} \\
& \left.+\frac{1}{2}\left(\partial_{\mu} \phi+g A_{\mu}\right)\left(\partial^{\mu} \phi+g A^{\mu}\right)+\frac{1}{2} g A^{2}\right] .
\end{aligned}
$$

It is not surprising therefore that a rigorous analysis of the Schwinger model dynamics based on (2.10) leads to difficulties, as (2.11) is clearly not Poincaré invariant. Equally serious however is the fact that $j^{\mu}(x)$ is not locally gauge invariant. This is a result of the same limiting process which led to the appearance of the Schwinger terms in (2.7). Instead the gauge transformations of the current operators follow from the additive transformation

$$
\phi(x) \sim \rightarrow \phi(x)-g \lambda(x)
$$

when $\psi(x) \sim \rightarrow e^{i \lambda(x)} \psi(x), \square \lambda(x)=0$. In order to make (2.11) both Poincaré and gauge invariant we must add a term of the form $-\frac{1}{2} g A^{2}$ to the integrand. Such an additional term is to be expected from the results of perturbation theory. If the photon self energy is evaluated in a Poincaré and gauge covariant way it is found to be non-zero, contrary to expectations. The result obtained is precisely of the form suggested above.

One could proceed now with a corrected cutoff Hamiltonian in place of (2.4) and attempt to establish the existence of the dynamics in the limit as the cutoff is removed (cf. [9]). For the Euclidean version of the model this has been discussed in [22]. This is not however, central to our purpose. Our interest lies in the algebraic structure of the model, and as we shall see, the boson form provides a shortcut to these aspects. We make one remark however. Although the classical Schwinger model is locally gauge invariant, the process of quantising the Fermi fields in the Fock representation of the CAR breaks this symmetry which must then be restored by the ad hoc addition of a counterterm. There is every reason to expect that this phenomenon is typical of the present constructive approach to field theory. It would obviously be desirable to develop a quantisation procedure which, like gauge invariant renormalisation in perturbation theory, leads unambiguously from the classical to the quantum model in a gauge invariant way. 
It is not clear that the Euclidean lattice approach to guage theories is such a procedure.

Returning to the Schwinger model, we note that the Fermi method with appropriate counterterm, gives a Poincaré and gauge invariant boson Hamiltonian. However, as we shall sketch in the next section, this Hamiltonian does not define an implementable dynamics in the Fock representation of the boson model. This problem does not arise in the Landau version. It is not necessary to repeat the foregoing discussion for the Landau Lagrangian as the only changes which need to be made are in the term $H_{A}$ (whereas the preceding concerned $H_{\psi}+H_{I}$ ).

With these preliminaries we shall now abandon the Schwinger model in its various forms and argue as follows. The fermion fields are unobservable, and insofar as the current and photon fields are concerned, their dynamics is contained in the boson Hamiltonian (2.10) and its modifications. Therefore we shall now treat the Schwinger model as a boson theory using a Poincare and gauge invariant Lagrangian and shall attempt to reconstruct the fermion fields from their algebraic properties with respect to the boson fields. We remark again that this equivalence of the Schwinger and boson dynamics is exact if cutoffs are introduced. We believe that this is sufficient evidence for concentrating on the bosonised form of the Schwinger model, particularly as we are primarily interested in investigating what happens to a Doplicher, Haag, Roberts analysis [12] in the presence of a local gauge symmetry.

\section{Linear Boson Models}

\subsection{Classical Solutions}

We will consider the two boson models arising from the Lagrangians (2.1a) and (2.1b). These models have Lagrangian densities

$$
\begin{aligned}
& \mathscr{L}_{1}=-\frac{1}{2} A_{\mu, v}\left(A^{\mu, v}-A^{v, \mu}\right)+\frac{1}{2} \partial^{\mu} \phi \partial_{\mu} \phi-\frac{1}{2}\left(\partial_{\mu} A^{\mu}\right)^{2}+\frac{1}{2} g^{2} A^{\mu} A_{\mu}+g A^{\mu} \partial_{\mu} \phi \\
& \mathscr{L}_{2}=-\frac{1}{2} A_{\mu, v}\left(A^{\mu, v}-A^{v, \mu}\right)+\frac{1}{2} \partial^{\mu} \phi \partial_{\mu} \phi+\frac{1}{2} g^{2} A^{\mu} A_{\mu}+\xi \partial_{\mu} A^{\mu}+g A^{\mu} \partial_{\mu} \phi
\end{aligned}
$$

respectively (we will write $x_{0} \equiv t, x^{1} \equiv x$, however for many purposes one can treat these as four dimensional models). The equations of motion are

and

$$
\left(\square+g^{2}\right) A_{\mu}=-g \partial_{\mu} \phi, \quad \square \phi=-g \partial^{\mu} A_{\mu}, \quad \square \partial^{\mu} A_{\mu}=0
$$

$$
\left(\square+g^{2}\right) A_{\mu}=\partial_{\mu} \eta, \quad \square \eta=\square \phi=0, \quad \partial^{\mu} A_{\mu}=0
$$

respectively, with

$$
\eta=\xi-g \phi .
$$

A brief calculation gives the most general real solutions of these equations of motion as follows.

For (3.3) we obtain

and

$$
\phi(t, x)=\beta(t, x)-i g t \chi(t, x)
$$

$$
A_{\mu}(t, x)=-\frac{1}{g} \partial_{\mu} \phi(t, x)-\frac{1}{g^{2}} \partial_{\mu}\left(\partial^{v} A_{v}(t, x)\right)+\alpha_{\mu}(t, x)
$$


where $\beta$ and $\alpha_{\mu}$ are solutions of $\square \beta=0$ and $\left(\square+g^{2}\right) \alpha_{\mu}=0$ while $\chi$ is defined by setting

$$
\chi(t, x)=\int_{-\infty}^{\infty} e^{i k x}\left(e^{-i \omega x} \hat{\gamma}(\omega, k)-\overline{\hat{\gamma}(\omega,-k)} e^{i \omega t}\right) d k / 4 \omega^{2}
$$

where $\omega=|k|$ and

$$
\partial^{\mu} A_{\mu}(t, x)=\int_{-\infty}^{\infty} e^{i k x}\left(e^{-i \omega t} \hat{\gamma}(\omega, k)-\overline{\hat{\gamma}(\omega,-k)} e^{i \omega t}\right) \frac{d k}{2 \omega} .
$$

For (3.4) the general solutions are

$$
A_{\mu}(t, x)=\alpha_{\mu}(t, x)+\frac{1}{g^{2}} \partial_{\mu} \eta(t, x)
$$

where

$$
\left(\square+g^{2}\right) \alpha_{\mu}=\square \phi=\square \eta=0
$$

and $\eta$ satisfies (3.5).

\subsection{Cauchy Problem}

Our aim is to obtain linear spaces of solutions, for each of these models, which are Poincaré invariant and possess Poincaré invariant symplectic forms. To do this we need to specify our initial data. Given solutions of (3.3) and (3.4) we will write the initial data as sextuples

and

$$
\Xi: x \rightarrow\left(A(0, x), \dot{A}(0, x), A_{0}(0, x), \dot{A}_{0}(0, x), \phi(0, x), \dot{\phi}(0, x)\right)
$$

$$
\Gamma: x \rightarrow\left(A(0, x), \dot{A}(0, x), A_{0}(0, x), \dot{\phi}(0, x), \phi(0, x), \eta(0, x)\right)
$$

respectively, where all the functions are smooth and of compact support on $\mathbb{R}$ (here we have set $A^{1}(0, x)=A(0, x)$ ). This initial data will be constrained to satisfy further conditions later. It is a simple matter to deduce from (3.1) and (3.2) the canonical variables of each model. We take our Poisson bracket (or symplectic form) to be given by the canonical expression. For example for (3.1) it is

$$
\begin{aligned}
& \int\left(A(0, x) \pi_{A^{\prime}}(0, x)-\pi_{A}(0, x) A^{\prime}(0, x)+A_{0}(0, x) \pi_{A_{0}}^{\prime}(0, x)-\pi_{A_{0}}(0, x) A_{0}^{\prime}(0, x)\right. \\
& \left.\quad+\phi(0, x) \pi_{\phi^{\prime}}(0, x)-\phi^{\prime}(0, x) \pi_{\phi}(0, x)\right) d x
\end{aligned}
$$

where

$$
\pi_{A^{\prime}}=\dot{A}^{\prime}+\nabla A_{0}^{\prime}, \quad \pi_{A_{0}^{\prime}}=-\dot{A}_{0}^{\prime}-\nabla A^{\prime}, \quad \pi_{\phi^{\prime}}=\dot{\phi}^{\prime}+g A_{0}^{\prime}
$$

(here $\nabla \equiv \frac{\partial}{\partial x}$. In terms of the initial data (3.10) and (3.11) we obtain the expressions:

$$
\begin{aligned}
B\left(\Xi, \Xi^{\prime}\right)=\int\left(\dot{\phi}(x) \phi^{\prime}(x)-\dot{\phi}^{\prime}(x) \phi(x)+\dot{A}_{0}^{\prime}(x) A_{0}(x)-\dot{A}_{0}(x) A_{0}^{\prime}(x)\right. \\
\left.+\dot{A}(x) A^{\prime}(x)-\dot{A}^{\prime}(x) A(x)-g \phi(x) A_{0}^{\prime}(x)+g \phi^{\prime}(x) A_{0}(x)\right) d x
\end{aligned}
$$

where $A(x) \equiv A(0, x)$ etc. 
and

$$
\begin{array}{r}
B\left(\Gamma, \Gamma^{\prime}\right)=\int\left[\dot{A}(x) A^{\prime}(x)-A(x) \dot{A}^{\prime}(x)+\nabla A(x) A_{0}^{\prime}(x)-\nabla A^{\prime}(x) A_{0}(x)\right. \\
\left.+\eta(x) A_{0}^{\prime}(x)-\eta^{\prime}(x) A_{0}(x)+\dot{\phi}(x) \phi^{\prime}(x)-\phi(x) \dot{\phi}^{\prime}(x)\right] d x
\end{array}
$$

respectively.

In order that the global gauge symmetry $\phi \rightarrow \phi+c(c \in \mathbb{R})$ of the Boson Lagrangians is not spontaneously broken in the representations of the various algebras we will be considering, we need to impose additional constraints on our initial data (cf. [23]). For (3.10) we require

$$
\int_{-\infty}^{\infty} \dot{\phi}(x) d x=0
$$

(which forces constraints on $\left.\partial^{\mu} A_{\mu}(0, x)\right)$ and for (3.11) we have

$$
\int_{-\infty}^{\infty} \dot{\xi}(x) d x=0
$$

in addition to (3.14). Denote by $M_{1}$ and $M_{2}$ respectively the real linear spaces spanned by the initial data (3.10) and (3.11). That the respective symplectic forms (3.12) and (3.13) are invariant under Poincare transformations is most easily verified by Fourier transforming the solutions (3.6) and (3.9). For both models we write

$$
\hat{\alpha}_{\mu}(k)=\frac{1}{\sqrt{2 \pi}} \int e^{-i k x}\left(\omega_{g} \alpha_{\mu}(x)+i \dot{\alpha}_{\mu}(x)\right) d x
$$

where

$$
\omega_{g}=\sqrt{\omega^{2}+g^{2}} \text { and } \quad \alpha_{\mu}(x) \equiv \alpha_{\mu}(0, x), \dot{\alpha}_{\mu}(x) \equiv \dot{\alpha}_{\mu}(0, x)
$$

We let

$$
\begin{gathered}
\hat{\eta}(k)=\frac{1}{\sqrt{2 \pi}} \int e^{-i k x}(\omega \eta(x)+i \dot{\eta}(x)) d x \\
\hat{\beta}(k)=\frac{1}{\sqrt{2 \pi}} \int e^{-i k x}(\omega \beta(x)+i \dot{\beta}(x)) d x,
\end{gathered}
$$

while for (3.9), $\square \phi=0$ so that

$$
\hat{\phi}(k)=\frac{1}{\sqrt{2 \pi}} \int e^{-i k x}(\omega \phi(x)+i \phi(x)) d x .
$$

However, for (3.6) we have $\phi(t, x)=\beta(t, x)-i g t \chi(t, x)$ and this leads eventually to the expression

$$
\begin{aligned}
\mathrm{B}\left(\Xi, \Xi^{\prime}\right)= & \frac{1}{i} \int d k g^{2}\left[\hat{\alpha}(k)^{*} \hat{\alpha}^{\prime}(k)-\hat{\alpha}(k) \hat{\alpha}^{\prime}(k)^{*}\right] / 2 \omega_{g}^{3} \\
& +\frac{1}{i} \int \frac{d k}{2 g \omega}\left[\hat{\beta}(k)^{*} \hat{\gamma}^{\prime}(k)-\hat{\beta}(k) \hat{\gamma}^{\prime}(k)^{*}+\hat{\gamma}(k)^{*} \hat{\beta}^{\prime}(k)-\hat{\gamma}(k) \hat{\beta}^{\prime}(k)^{*}\right]
\end{aligned}
$$




$$
+\frac{1}{i} \int d k\left[\left(\frac{1}{2 \omega g^{2}}+\frac{1}{4 \omega 3}\right)\left(\hat{\gamma}(k)^{*} \hat{\gamma}^{\prime}(k)-\hat{\gamma}(k) \hat{\gamma}^{\prime}(k)^{*}\right)\right] .
$$

In the case of (3.13) we obtain

$$
\begin{aligned}
B\left(\Gamma, \Gamma^{\prime}\right)= & \frac{1}{i} \int d k g^{2}\left[\hat{\alpha}(k)^{*} \hat{\alpha}^{\prime}(k)-\hat{\alpha}(k) \hat{\alpha}^{\prime}(k)^{*}\right] / 2 \omega_{g}^{3} \\
& +\frac{1}{i} \int d k\left\{\left[\hat{\phi}(k)^{*} \hat{\phi}^{\prime}(k)-\hat{\phi}(k) \hat{\phi}^{\prime}(k)^{*}\right]\right. \\
& \left.+\frac{1}{g^{2}}\left[\hat{\eta}(k) \hat{\eta}^{\prime}(k)^{*}-\eta(k)^{*} \hat{\eta}^{\prime}(k)\right]\right\} / 2 \omega .
\end{aligned}
$$

Here we have used $\dot{\alpha}_{0}(x)=-\nabla \alpha(x)$ to simplify the expressions.

Now a tedious but straightforward calculation shows that (3.15) is Poincare invariant while the Poincaré invariance of (3.16) is clear.

Note that the Poincaré group acts on solutions of (3.2) and (3.4) in the obvious way ([6] contains the details). That $M_{1}$ and $M_{2}$ are themselves Poincaré invariant needs some calculation, in particular the invariance of (3.14) is not immediately apparent (a similar calculation appears however in [23]). In the case of $M_{1}$, Poincare invariance forces constraints on the functions $\partial^{\mu} A_{\mu}(x)$, however the details are not relevant for the subsequent discussion so we omit them.

Now we have the necessary structure to define a $C^{*}$ algebra for the CCR over $M_{1}$ and $M_{2}$ in the usual way (cf. [16] and [24]). In the case of $M_{2}$ this is done in some detail in [6]. To complete this discussion we need to find a complex structure on $M_{1}$ and $M_{2}$ which makes them complex pre-Hilbert spaces and also defines the Fock representation of the corresponding $C^{*}$-algebras. For $M_{1}$ we let

$$
J_{F}^{1} \hat{\alpha}(k)=i \hat{\alpha}(k) ; \quad J_{F}^{1} \hat{\beta}(k)=i \hat{\beta}(k) ; \quad J_{F}^{1} \hat{\lambda}(k)=i \hat{\lambda}(k)
$$

where $\hat{\lambda}(k)=\hat{\beta}(k)+\left(\frac{1}{g}+\frac{g}{4 \omega^{3}}\right) \hat{\gamma}(k)$; giving the positive definite inner product:

$$
\left\langle\Xi, \Xi^{\prime}\right\rangle=B\left(\Xi, J \frac{1}{F} \Xi^{\prime}\right)+i B\left(\Xi, \Xi^{\prime}\right)
$$

For $M_{2}$ we set

$$
J_{F} \hat{\alpha}(k)=i \hat{\alpha}(k) ; \quad J_{F} \hat{\phi}(k)=i \hat{\phi}(k) ; \quad J_{F} \hat{\eta}(k)=-i \hat{\eta}(k) ;
$$

with a corresponding inner product $\left\langle\Gamma, \Gamma^{\prime}\right\rangle$ defined as for $M_{1}$. There are a number of important observations to be made at this point.

1. In both cases there is a natural complex structure on $M_{1}$ and $M_{2}$ which is suggested by the canonical Hamiltonian formalism. This differs from (3.17) and (3.19) in being multiplication by $+i$ for every field. This complex structure leads to an indefinite inner product on both $M_{1}$ and $M_{2}$. If we were to pursue the indefinite metric programme [14], [25] we could quantise these models in an indefinite metric space.

2. We denote by $\Delta_{c}\left(M_{1}\right)$ and $\Delta_{c}\left(M_{2}\right)$ the CCR algebras over $M_{1}$ and $M_{2}$ 
(these are discussed in more detail in the next section). Then the complex structures (3.17) and (3.19) define the Fock representations of these algebras in the usual way [24]. However in the case of (3.19), $J_{F}$ commutes with Poincare transformations on $M_{2}$ so that the Poincare group is unitarily implemented in the Fock representation. It is not difficult to verify that $J_{F}^{1}$ does not commute with the action of time translations (or boosts) on $M_{1}$. To see this observe that $J_{F}^{1} \hat{\gamma}(k)=$ $i \hat{\gamma}(k)+2 i \hat{\beta}(k) / \varepsilon(\omega)$ where $\varepsilon(\omega)=\frac{1}{g}+\frac{g}{4 \omega^{3}}$ while the time evolution is given by the one parameter group $t \rightarrow T_{t} t \in \mathbb{R}$, where

$$
\begin{gathered}
T_{t} \hat{\alpha}(k)=e^{-i \omega t} \hat{\alpha}(k), \quad T_{t} \hat{\beta}(k)=(\hat{\beta}(k)-i g t \hat{\gamma}(k)) e^{-i \omega t} \\
T_{t} \hat{\gamma}(k)=e^{-i \omega t} \hat{\gamma}(k) .
\end{gathered}
$$

It is easy to demonstrate that the only complex structure which commutes with (3.20) is the canonical indefinite metric one. A case by case analysis of quadratic Hamiltonians [26] shows that this is not a typical. (In fact there appears to be a general no-go theorem which restricts the application of Segal's Weyl algebra formalism).

3. An analysis of the indefinite metric quantisation of the $M_{1}$ model reveals that the Hamiltonian is the sum of two commuting operators, one with a discrete spectrum and one with a continuous spectrum ranging from 0 to $\infty$.

\subsection{Field Algebras}

With the structure of the previous section we may define the $C^{*}$-algebra $\Delta_{c}\left(M_{1}\right)$ and $\Delta_{c}\left(M_{2}\right)$ as the CCR algebras over $M_{1}$ and $M_{2}$. We use the notation:

$$
\delta_{\Xi}: M_{1} \rightarrow \mathbb{R}, \quad \delta_{\Gamma}: M_{2} \rightarrow \mathbb{R}
$$

to denote the elements of $\Delta_{c}\left(M_{1}\right)$ and $\Delta_{c}\left(M_{2}\right)$ respectively defined by

$$
\delta_{\Xi}\left(\Xi^{\prime}\right)=\left\{\begin{array}{ll}
1 & \Xi=\Xi^{\prime} \\
0 & \Xi \neq \Xi^{\prime}
\end{array} \in M_{1} ; \quad \delta_{\Gamma}\left(\Gamma^{\prime}\right)=\left\{\begin{array}{ll}
1 & \Gamma=\Gamma^{\prime} \\
0 & \Gamma \neq \Gamma^{\prime}
\end{array} \in M_{2}\right.\right.
$$

with

$$
\delta_{\Xi^{\prime}} \delta_{\Xi^{\prime}}=\exp \frac{i}{2} B\left(\Xi, \Xi^{\prime}\right) \delta_{\Xi+\Xi^{\prime}}
$$

and a similar multiplication for $\Delta_{c}\left(M_{2}\right)$ (cf. [16] and [24]). As we mentioned in the previous section, in the Fock representation $\pi_{F}^{1}$ of $\Delta_{c}\left(M_{1}\right)$ specified by the generating functional

$$
\Xi \rightarrow \exp -\frac{1}{4} B\left(\Xi, J_{F}^{1} \Xi\right),
$$

Poincare transformations are not unitarily implemented. For the Fock representation $\pi_{F}^{2}$ of $\Delta_{c}\left(M_{2}\right)$, Poincaré transformations are implemented but the generator of time translations is not a positive operator. This is the price we pay for choosing a definite metric quantisation. We take the view that $\Delta_{c}\left(M_{1}\right)$ and $\Delta_{c}\left(M_{2}\right)$ are field algebras. That they have unphysical properties in the Fock representation 
appears to be an inevitable consequence of the presence of zero mass fields in the model. We shall define however, algebras of observables which, for both $\Delta_{c}\left(M_{1}\right)$ and $\Delta_{c}\left(M_{2}\right)$, satisfy all the Wightman axioms in an appropriate representation.

To define observable fields we need some criterion. We take the view that observables must be locally gauge invariant. To see what this means we need to define local gauge transformations on the algebras $\Delta_{c}\left(M_{1}\right)$ and $\Delta_{c}\left(M_{2}\right)$. We will do this in detail only for $\Delta_{c}\left(M_{2}\right)$. The case of $\Delta_{c}\left(M_{1}\right)$ follows similarly.

As in [6], we introduce certain subspaces:

$$
\begin{aligned}
& M_{\alpha}=\left\{\Gamma \in M_{2} \text { of form }\left(\alpha, \dot{\alpha}, \alpha_{0}, 0,0,0\right)\right\}, \\
& M_{\phi}=\left\{\Gamma \in M_{2} \text { of form }(0,0,0, \phi, \dot{\phi}, 0)\right\} \text {, } \\
& M_{\eta}=\left\{\Gamma \in M_{2} \text { of form }\left(\frac{1}{g^{2}} \nabla \eta, \frac{1}{g^{2}} \nabla \dot{\eta},-\frac{1}{g^{2}} \dot{\eta}, 0,0,-\eta\right)\right\} \text {, } \\
& M_{\xi}=\left\{\Gamma \in M_{2} \text { of form }\left(\nabla \xi, \nabla \dot{\xi},-\dot{\xi}, g \xi, g \dot{\xi},-g^{2} \xi\right)\right\} \text {. }
\end{aligned}
$$

Restricting the time zero initial data to these subspaces defines $C^{*}$-subalgebras $\Delta_{c}\left(M_{\alpha}\right), \Delta_{c}\left(M_{\phi}\right), \Delta_{c}\left(M_{\eta}\right)$ and $\Delta_{c}\left(M_{\xi}\right)$ respectively, of $\Delta_{c}\left(M_{2}\right)$. The quantised versions of the classical fields are defined by setting (with $\pi_{F}^{2}=\pi_{F}$ )

$$
R(\Gamma)=\left.\frac{1}{i} \frac{d}{d \lambda} \pi_{F}\left(\delta_{\lambda \Gamma}\right)\right|_{\lambda=0}
$$

for $\Gamma \in M_{2}$, and then restricting $\Gamma$ to lie in the appropriate subspace of $M_{2}$.

Corresponding to each $\Gamma_{\xi} \in M_{\xi}$ there is an automorphism $\alpha_{\xi}$ of $\Delta_{c}\left(M_{2}\right)$ given by

$$
\alpha_{\xi}\left(\delta_{\Gamma}\right)=\exp i B\left(\Gamma, \Gamma_{\xi}\right) \cdot \delta_{\Gamma} .
$$

This is the transformation of the quantised fields corresponding to the classical gauge transformation $\phi \rightarrow \phi+g \widetilde{\xi}, A_{\mu} \rightarrow A_{\mu}-\partial_{\mu} \xi$ where $\widetilde{\xi}$ is the solution of $\square \tilde{\xi}=0$ with initial data $\xi, \dot{\xi}$. The Lagrangian (3.2) is invariant under these gauge transformations and this is reflected in the quantised theory by the fact that (3.21) is an automorphism of $\Delta_{c}\left(M_{2}\right)$. The gauge group $\mathscr{G}$ is defined to be the group of all local gauge automorphisms $\alpha_{\xi}$ as $\Gamma_{\xi}$ ranges over $M_{\xi}$.

Now define the gauge invariant algebra as the $C^{*}$-subalgebra of $\Delta_{c}\left(M_{2}\right)$ which is fixed by the action of $\mathscr{G}$. This algebra is necessarily generated by those $\delta_{\Gamma} \in \Delta_{c}\left(M_{2}\right)$ for which $B\left(\Gamma, \Gamma_{\xi}\right)=0$ for all $\Gamma_{\xi} \in M_{\xi}$. A brief calculation shows that this is precisely the algebra $\Delta_{c}\left(M_{\alpha} \oplus M_{\xi}\right)$ (i.e. the completion in $\Delta_{c}\left(M_{2}\right)$ of the algebra generated by the $\delta_{\Gamma}$ with $\Gamma \in M_{\alpha} \oplus M_{\xi}$ ).

In the classical model one would expect that the conserved Noether current (arising from the invariance of the Lagrangian under the global gauge transformation $\phi \rightarrow \phi+c, c \in \mathbb{R})$ should be observable. In the quantised theory therefore we would expect to find an operator-valued distribution $f \rightarrow J(f)$ (where $f: \mathbb{R}^{2} \rightarrow$ $\mathbb{R}^{2}$ is smooth of compact support) which could be interpreted as the quantum analogue of the classical Noether current :

$$
j_{\mu}(x)=g \alpha_{\mu}(x)+\frac{1}{g} \partial_{\mu} \xi(x) .
$$


If for the moment we write (by an abuse of notation)

$$
J(f)=g \alpha_{\mu}\left(f^{\mu}\right)+\frac{1}{g} \xi\left(\partial^{\mu} f_{\mu}\right)
$$

interpreting $\alpha_{\mu}$ and $\xi$ as the quantised versions (in the Fock representation) of the corresponding classical fields, then it is a simple matter to show that there is an element $\Gamma_{f} \in M_{\alpha} \oplus M_{\xi}$ such that

$$
J(f)=\left.\frac{1}{i} \frac{d}{d \lambda} \pi_{F}\left(\delta_{\lambda \Gamma_{f}}\right)\right|_{\lambda=0}
$$

(this calculation appears in [6]). Furthermore as $f$ ranges over the test function space, so $\Gamma_{f}$ ranges over $M_{\alpha} \oplus M_{\xi}$.

Thus if we regard the quantised Noether current as observable then the gauge invariant algebra and the algebra of observables coincide. We note the following facts. :

1. The gauge automorphisms (3.21) are implemented in the Fock representation of $\Delta_{c}\left(M_{2}\right)$ via

$$
\begin{gathered}
\pi_{F}\left(\alpha_{\xi}\left(\delta_{\Gamma}\right)\right)=\pi_{F}\left(\delta_{\Gamma_{\xi}}\right) \pi_{F}\left(\delta_{\Gamma}\right) \pi_{F}\left(\delta_{\Gamma_{\xi}}\right)^{-1} \\
\delta_{\Gamma_{\xi}} \in \Delta_{c}\left(M_{\xi}\right) .
\end{gathered}
$$

2. $\pi_{F}\left(\Delta_{c}\left(M_{\xi}\right)\right)^{\prime \prime}$ is an abelian von Neumann algebra and

$$
\pi_{F}\left(\Delta_{c}\left(M_{\alpha} \oplus M_{\xi}\right)\right) \subseteq \pi_{F}\left(\Delta_{c}\left(M_{\xi}\right)\right)^{\prime} .
$$

In [6] we showed that $\pi_{F}\left(\Delta_{c}\left(M_{\xi}\right)\right)^{\prime \prime}$ determines a direct integral decomposition of the Fock space and hence a direct integral decomposition of the representation $\pi_{F} \uparrow \Delta_{c}\left(M_{\alpha} \oplus M_{\xi}\right)$ of the gauge invariant algebra. The representations occurring in this decomposition are all irreducible.

3. An analysis similar to the above goes through for $\Delta_{c}\left(M_{1}\right)$. However we feel that it is necessary to find a time translation invariant pure state on $\Delta_{c}\left(M_{1}\right)$ before it could be regarded as a physically reasonable field algebra.

4. There is a local structure on the field algebras $\Delta_{c}\left(M_{1}\right)$ and $\Delta_{c}\left(M_{2}\right)$ determined by taking, for each double cone $\mathcal{O} \subseteq \mathbb{R}^{2}$, the algebras $\Delta_{c}\left(M_{1}(\mathcal{O})\right.$ ) (respectively $\Delta_{c}\left(M_{2}(\mathcal{O})\right)$ ) to be generated by the $\delta_{\Xi}$ (respectively $\delta_{\Gamma}$ ) where the solution to the field equations determined by $\Xi$ (respectively $\Gamma$ ) has initial data on some space like hyperplane $P$ with support in $P \cap \mathcal{O}$. The various subalgebras of $\Delta_{c}\left(M_{2}\right)$ defined in this section inherit this local structure in the obvious way. In particular the algebra $\Delta_{c}\left(M_{\xi}\right)$ generated by the gauge transformations has a local structure.

5. The conditions (3.14) and (3.14') are suggested by the analogous condition in [23]. They arise because it is $\partial_{\mu} \phi$ which is of importance in the Schwinger model (being the free current) not $\phi$. They also ensure that the infrared divergence associated with massless fields in 2 dimensions does not cause difficulties here. Finally they allow us to avoid the vacuum degeneracy which preoccupies the indefinite metric approach [27] (a degeneracy we regard as unphysical). 
For the remainder of the paper we will refer to $\pi_{F} \uparrow_{c}\left(M_{\alpha} \oplus M_{\xi}\right)$ as the Fock representation of the gauge invariant algebra. We record the following result (whose proof appears in [6]) for later use

Lemma. $\pi_{F} \uparrow \Delta_{c}\left(M_{\alpha} \oplus M_{\xi}\right)$ is cyclic (with cyclic vector equal to the Fock vacuum).

\subsection{Observables}

As we remarked above, the gauge invariant algebra is observable if we assume that the classical Noether current should have an observable quantum analogue. This leads us to consider the Fock representation of the gauge invariant algebra in which the generator of time translations is not positive. The question therefore arises: what are the physical states of the theory? It is not difficult to write down irreducible representations of $\Delta_{c}\left(M_{\alpha} \oplus M_{\xi}\right)$ in which time translations are implemented. As $\Delta_{c}\left(M_{\xi}\right)$ is central in $\Delta_{c}\left(M_{\alpha} \oplus M_{\xi}\right)$, it will be represented by scalars and so such representations reduce essentially to representations of the massive boson algebra $\Delta_{c}\left(M_{\alpha}\right)$ and here positivity of the energy is easily ensured. Hence a natural class of physical states on $\Delta_{c}\left(M_{\alpha} \oplus M_{\xi}\right)$ may be obtained in this way.

There is a difficulty with considering only these states. Since we are in the business of constructing the Schwinger model and not just the superselection sectors of a boson model, we must reconstruct a Hilbert space on which fermion operators may be defined. In order to do this we are forced to begin with non-irreducible representations of $\Delta_{c}\left(M_{\alpha} \oplus M_{\xi}\right)$. Moreover $\pi_{F}$ appears to be the "minimal" representation of $\Delta_{c}\left(M_{\alpha} \oplus M_{\xi}\right)$ which will serve. Thus we are forced back into the difficulty with negative energies.

Now the fields giving rise to these negative energies may be dynamically decoupled from the other fields and hence no transitions to negative energy states are possible. The Schwinger model is just a little too unphysical for us to decide whether this resolution of the difficulty is acceptable. We nevertheless believe that a definite metric approach to $(\mathrm{QED})_{4}$ in which the full 4-component vector potential is quantised (and hence local gauge transformations implemented) will have to overcome a similar difficulty.

We therefore favour the following line of argument.

Although only positive energy states on the observable algebra are physically acceptable (and hence the only observable field in the Schwinger model will be the massive boson field) the dynamics of the observable algebra are derived from a representation in which local gauge automorphisms (and hence fermion operators) are defined. This representation produces a non-positive generator of time translations, in which the "negative energy fields" evolve independently of the rest of the field algebra, and hence produce no observable effects. This is the price necessary to pay for avoiding an indefinite metric, and whether it is too big will only become clear when less trivial models are analysed.

Henceforth we will therefore reserve observable for the boson algebra $\Delta_{c}\left(M_{\alpha}\right)$ and in the next Section analyse the sectors arising from the gauge invariant algebra $\Delta_{c}\left(M_{\alpha} \oplus M_{\xi}\right)$. This raises the question of how one might try to construct candidates for a gauge invariant algebra given only $\Delta_{c}\left(M_{\alpha}\right)$ and the gauge group $M_{\xi}$. 
Although this is peripheral to our main purpose we will sketch a line of argument here. We note firstly that $\Delta_{c}\left(M_{\alpha} \oplus M_{\xi}\right) \cong \Delta_{c}\left(M_{\alpha}\right) \otimes \Delta_{c}\left(M_{\xi}\right)$ (this follows directly from the fact that $\Delta_{c}\left(M_{2}\right) \cong \Delta_{c}\left(M_{\alpha}\right) \otimes \Delta_{c}\left(M_{\eta} \oplus M_{\xi}\right)$, cf.Manuceau [16]) and that the sequence $\Delta_{c}\left(M_{\xi}\right) \rightarrow \Delta_{c}\left(M_{\alpha} \oplus M_{\xi}\right) \rightarrow \Delta_{c}\left(M_{\alpha}\right)$ is exact. It would appear therefore that we are looking for extensions of $\Delta_{c}\left(M_{\xi}\right)$ by $\Delta_{c}\left(M_{\alpha}\right)$ and the question is therefore whether the tensor product $\Delta_{c}\left(M_{\alpha}\right) \otimes \Delta_{c}\left(M_{\xi}\right)$ is the only acceptable one. As stated this is a rather difficult problem. If however we restrict attention to those extensions which arise from the underlying abelian groups $M_{\alpha}$ and $M_{\xi}$, then we can obtain a reasonably satisfactory answer. (In practice such extensions are probably the only ones that could be written down.) Such extensions are determined by 2 cocycles $\sigma: M_{\alpha} \times M_{\alpha} \rightarrow M_{\xi}$ satisfying

$$
\sigma\left(\Xi_{\alpha}, \Xi_{\alpha^{\prime}}\right)+\sigma\left(\Xi_{\alpha}+\Xi_{\alpha^{\prime}}, \Xi_{\alpha^{\prime \prime}}\right)=\sigma\left(\Xi_{\alpha^{\prime}}, \Xi_{\alpha^{\prime \prime}}\right)+\sigma\left(\Xi_{\alpha}, \Xi_{\alpha^{\prime}}+\Xi_{\alpha^{\prime \prime}}\right) .
$$

Since we require $\lambda \rightarrow \pi\left(\delta_{\lambda \Xi_{\alpha}}\right)$ to be a one parameter unitary group in physically acceptable representations, we need to impose the condition that $\sigma$ be bilinear. Moreover if the generator of $\lambda \rightarrow \pi\left(\delta_{\lambda \Sigma_{\alpha}}\right)$ is to be an operator valued distribution then $\sigma$ will need to be continuous in the Schwartz topology on the product $M_{\alpha} \times M_{\alpha}$ of the initial data space $M_{\alpha}$. It is now not difficult using the (Schwartz) kernel theorem to write down many such cocycles. There is however one further requirement, namely that the actions of the Poincare group on $M_{\alpha}$ and $M_{\xi}$ extend to automorphisms of the extension. This forces the condition:

$$
(a, \Lambda) . \sigma\left(\Xi_{\alpha}, \Xi_{\alpha^{\prime}}\right)=\sigma\left((a \cdot \Lambda) \Xi_{\alpha},(a, \Lambda) \Xi_{\alpha^{\prime}}\right)
$$

for Poincare transformations $(a, \Lambda)$. Writing out this equation in terms of kernels in the case of time translations and differentiating, immediately leads to the conclusion that $\sigma\left(\Xi_{\alpha}, \Xi_{\alpha^{\prime}}\right)=0$ for all $\Xi_{\alpha}, \Xi_{\alpha^{\prime}}$. So in terms of group extensions, the direct sum $M_{\alpha} \oplus M_{\xi}$ is the only acceptable solution to the problem.

\section{Reconstructing the Schwinger Model}

\subsection{Streater-Wilde Sectors}

Here we apply the analysis of Streater and Wilde [23] to the gauge invariant subalgebra of $\Delta_{c}\left(M_{2}\right)$. We firstly recover the charge sectors of Lowenstein and Swieca [18] for the Schwinger model using local automorphisms of the gauge invariant algebra.

Let $\theta$ be a solution of the wave equation, $\square \theta=0$, such that the initial data $x \rightarrow(\theta(0, x), \dot{\theta}(0, x))$ are $C^{\infty}$ and $x \rightarrow \dot{\theta}(0, x)$ is of compact support. Then there is an automorphism $\gamma_{\theta}$ of $\Delta_{c}\left(M_{\alpha} \oplus M_{\xi}\right)$ given by

where

$$
\gamma_{\theta}\left(\delta_{\Gamma_{\alpha}+\Gamma_{\xi}}\right)=\exp i B_{0}\left(\theta, \Gamma_{\xi}\right) \cdot \delta_{\Gamma_{\alpha}+\Gamma_{\xi}}
$$

$$
B_{0}\left(\theta, \Gamma_{\xi}\right)=g \int \theta(0, x) \dot{\xi}(0, x)-\dot{\theta}(0, x) \xi(0, x) d x
$$

If $\mathcal{O} \subseteq \mathbb{R}^{2}$ is a double cone then by choosing a solution $\tilde{\eta}$ of the wave equation 
such that $g \tilde{\eta} \uparrow \mathcal{O}=\theta \uparrow \mathcal{O}$ and letting

$$
\Gamma_{\eta}=\frac{1}{2}(0,0,0,-g \eta,-g \dot{\eta}, 0)
$$

where $\eta, \dot{\eta}$ are the initial data of $\tilde{\eta}$ we see that $\gamma_{\theta}$ is implemented (as an automorphism of the local algebra $\Delta_{c}\left(M_{\alpha}(\mathcal{O})+M_{\xi}(\mathcal{O})\right)$ by $\pi_{F}\left(\delta_{\Gamma_{\eta}}\right)$ in the Fock representation

The properties of these automorphisms are summarised in the following proposition whose proof is identical to that of the corresponding results in Streater and Wilde [23] (see also Streater [28]).

Proposition 4.1. (i) The automorphisms (4.1) are implemented in $\pi_{F}$ if and only if $\theta(-\infty)=\theta(\infty)$ and $\int \dot{\theta}(x)=0$.

(ii) The representation $\pi_{\theta}=\pi_{F}^{\circ} \gamma_{\theta}$ of $\Delta_{c}\left(M_{\alpha} \oplus M_{\xi}\right)$ is strongly locally equivalent to $\pi_{F} \uparrow \Delta_{c}\left(M_{\alpha} \oplus M_{\xi}\right)$.

(iii) Each automorphism $\gamma_{\theta}$ is localised in the sense of [12].

We note also that the conditions stated in [23] for the equivalence of two representations $\pi_{\theta_{1}}, \pi_{\theta_{2}}$, corresponding to distinct automorphisms $\gamma_{\theta_{1}}, \gamma_{\theta_{2}}$ also apply here.

Now by the Lemma at the end of the previous section $\pi_{\theta}$ is cyclic and so there is a G.N.S. Hilbert space $\mathscr{H}\left(\pi_{\theta}\right)$ on which $\pi_{\theta}$ acts. We introduce the operator $W(\theta): \mathscr{H}\left(\pi_{F}\right) \rightarrow \mathscr{H}\left(\pi_{\theta}\right)$ defined by

$$
W(\theta) \pi_{F}\left(\delta_{\Gamma}\right) \Omega_{F}=\exp i B_{0}(\theta, \Gamma) \pi_{\theta}\left(\delta_{\Gamma}\right) \Omega_{\theta}
$$

where $\Omega_{\theta}$ is the cyclic vector in $\mathscr{H}\left(\pi_{\theta}\right)$. Then the definition of $W(\theta)$ may be extended so that it may be regarded as an operator from $\mathscr{H}\left(\pi_{\theta_{1}}\right)$ to $\mathscr{H}\left(\pi_{\theta+\theta_{1}}\right)$ for any representation $\pi_{\theta_{1}}=\pi_{F} \circ \gamma_{\theta_{1}}$ arising in the above way. Specifically we write

$$
W(\theta) \pi_{\theta_{1}}\left(\delta_{\Gamma}\right) \Omega_{\theta_{1}}=\exp \left[-\frac{i}{2} B_{0}\left(\theta, \theta_{1}\right)+i B_{0}(\theta, \Gamma)\right] \pi_{\theta+\theta_{1}}\left(\delta_{\Gamma}\right) \Omega_{\theta+\theta_{1}}
$$

where

$$
B_{0}\left(\theta, \theta_{1}\right)=\int\left[\theta(0, x) \dot{\theta}_{1}(0, x)-\dot{\theta}(0, x) \theta_{1}(0, x)\right] d x .
$$

Consequently

$$
W(\theta) W\left(\theta_{1}\right)=e^{-i B_{0}\left(\theta, \theta_{1}\right)} W\left(\theta_{1}\right) W(\theta) .
$$

By a suitable choice of $\theta, \theta_{1}$ it is possible to have anticommuting operators in the model as in [23].

We refer to the representations $\pi_{\theta}$ as Streater-Wilde sectors. These sectors are "charged" and the charges are determined by the conserved currents of the bare Schwinger model (namely $\partial_{\mu} \phi$ and $\varepsilon_{\mu \nu} \partial^{v} \phi$ ). These charges may be defined as follows (cf. Streater [29]). Each solution $\theta$ of the wave equation may be decomposed as

$$
\theta(t, x)=f(x+t)+g(x-t)
$$


(the decomposition is Poincare invariant). We define

$$
Q_{+}(\theta)=(f(\infty)-f(-\infty)), \quad Q_{-}(\theta)=-(g(\infty)-g(-\infty)) .
$$

Without loss of generality we may suppose that $f(-\infty)=g(-\infty)=0$. Hence we let

$$
\begin{aligned}
\int_{-\infty}^{\infty} \dot{\theta}(x) d x & =\left(Q_{+}(\theta)+Q_{-}(\theta)\right) \stackrel{\text { def }}{=} Q(\theta) \\
\theta(0, \infty) & =\left(Q_{+}(\theta)-Q_{-}(\theta)\right) \stackrel{\text { def }}{=} Q_{5}(\theta)
\end{aligned}
$$

and $Q(\theta)$ and $Q_{5}(\theta)$ are the charges of the sectors $\pi_{\theta}$.

Our eventual aim is to produce a Hilbert space on which Fermion operators may be defined. To do this we combine the above treatment of sectors with the discussion of Streater [28] on the Thirring model and the analysis of Dell' Antonio, Frischmann and Zwanziger [29].

Fix a $C^{\infty}$-function of compact support in $\mathbb{R}$, say $\dot{\eta}$ and define

$$
\eta(x)=\int_{-\infty}^{x} \dot{\eta}(x) d x .
$$

Choose $\dot{\eta}$ so that $\eta(\infty)=1$. Now the solution of the wave equation having initial data $(\sqrt{\pi \eta}, \sqrt{\pi \dot{\eta}})$ is a function $\tilde{\eta}_{+}$of $t+x$. Similarly the solution $\tilde{\eta}_{-}$of the wave equation having initial data $(-\sqrt{\pi \eta}, \sqrt{\pi \dot{\eta}})$ is a function of $x-t$. We write $\gamma_{\eta_{+}}$ and $\gamma_{\eta_{-}}$for the automorphisms of $\Delta_{c}\left(M_{\alpha} \oplus M_{\xi}\right)$ defined by these solutions. The space $\mathscr{H}_{m, n}$ is defined to be the GNS Hilbert space carrying the representation $\pi_{F}{ }^{\circ} \gamma_{m \eta_{+}+n \eta_{-}}$of $\Delta_{c}\left(M_{\alpha} \oplus M_{\xi}\right)$. Form the direct sum

$$
\mathscr{H}=\bigotimes_{m, n=-\infty}^{\infty} \mathscr{H}_{m, n} .
$$

We will see in the next section that Fermion fields may be defined on $\mathscr{H}$. Notice that on $\mathscr{H}_{m, n}, Q=(m+n) \sqrt{\pi}$ and $Q_{5}=(m-n) \sqrt{\pi}$. There are two charge shift operators defined on $\mathscr{H}$, namely $W\left(\tilde{\eta}_{+}\right)$and $W\left(\tilde{\eta}_{-}\right)$, which as a consequence of (4.2) and (4.3) satisfy

$$
W\left(\tilde{\eta}_{+}\right) W\left(\tilde{\eta}_{-}\right)=-W\left(\tilde{\eta}_{-}\right) W\left(\tilde{\eta}_{+}\right) .
$$

These operators represent the charge changing aspect of the Fermions.

\subsection{Reconstructing the Field Algebra}

In the preceding section we obtained a number of inequivalent "charged" sectors of the gauge invariant algebra. In this section we wish to make a number of observations on the problem of determining the field algebra given the gauge invariant algebra and these different sectors.

Firstly we note that the preceding analysis, although based on regarding $\Delta_{c}\left(M_{\alpha} \oplus M_{\xi}\right)$ as a subalgebra of $\Delta_{c}\left(M_{2}\right)$, could equally have been carried through if $\Delta_{c}\left(M_{\alpha} \oplus M_{\xi}\right)$ were thought of as the gauge invariant subalgebra of $\Delta_{c}\left(M_{1}\right)$. (It is not difficult to show that the gauge invariant subalgebra of $\Delta_{c}\left(M_{1}\right)$ is indeed 
isomorphic to $\left.\Delta_{c}\left(M_{\alpha} \oplus M_{\xi}\right)\right)$. Thus there are two states on $\Delta_{c}\left(M_{\alpha} \oplus M_{\xi}\right)$ determined by the Fock representations of $\Delta_{c}\left(M_{1}\right)$ and $\Delta_{c}\left(M_{2}\right)$. The discussion of the preceding section shows that the former is not time translation invariant while the latter is, thereby leading us to prefer $\Delta_{c}\left(M_{2}\right)$ over $\Delta_{c}\left(M_{1}\right)$ as a field algebra. We remark in this direction that the elements $\delta_{\Gamma_{\eta}}$ of $\Delta_{c}\left(M_{2}\right)$ define transportable automorphisms (in the sense of Roberts [30] of $\Delta_{c}\left(M_{\alpha} \oplus M_{\xi}\right)$ via

$$
\delta_{\Gamma_{\alpha}+\Gamma_{\xi}} \mapsto \exp i B\left(\Gamma_{\eta}, \Gamma_{\xi}\right) \delta_{\Gamma_{\alpha}+\Gamma_{\xi}} .
$$

(These automorphisms are of course implemented in the Fock representation of $\left.\Delta_{c}\left(M_{\alpha} \oplus M_{\xi}\right)\right)$. It would be interesting to know whether the local 1-cohomology of Roberts [31] serves, in the context of abelian gauge theories, to parametrize in some sense, the dual of the local gauge group.

One final observation we can make on this is that the direct integral decomposition of the Fock representation of $\Delta_{c}\left(M_{\alpha} \oplus M_{\xi}\right)$, determined by the abelian von Neumann algebra $\pi_{F}\left(\Delta_{c}\left(M_{\xi}\right)\right)^{\prime \prime}$ decomposes the state:

$$
\sigma_{F}: \delta_{\Gamma} \rightarrow \exp \left(-\frac{1}{4} B\left(\Gamma, J_{F} \Gamma\right)\right)
$$

into a direct integral of states $[6]$ :

$$
\sigma_{\zeta}: \delta_{\Gamma_{\alpha}+\Gamma_{\xi}} \rightarrow \zeta\left(\delta_{\Gamma_{\xi}}\right) \exp -\frac{1}{4} B\left(\Gamma_{\alpha}, J_{F} \Gamma_{\alpha}\right)
$$

where $\zeta$ ranges over the spectrum of $\Delta_{c}\left(M_{\xi}\right)$. The localised automorphisms $\gamma_{\theta}$ of the previous section define elements of the spectrum of $\Delta_{c}\left(M_{\xi}\right)$ via

$$
\Gamma_{\xi} \sim \exp \frac{i}{2} B\left(\theta, \Gamma_{\xi}\right) .
$$

Thus the "charged" sectors are "embedded" in the spectrum of the gauge group (a possibility suggested to us by Roberts [32]).

Turning now to the definition of the Fermion operators we note firstly that it would be interesting to see whether some sort of algebraic characterisation could be found for them. As we do not have one, we use the following ad hoc construction. Following [28] and $[29]^{1}$ we define an approximate $\delta$-function by

Now define

$$
\delta_{\Lambda}(x)=\frac{1}{2 \pi} \int_{|p|<\Lambda} e^{i p x} d p, \quad \Lambda>0 .
$$

$$
\theta_{\Lambda}(x)=\int_{-\infty}^{x} \delta_{\Lambda}(u) d u
$$

Let $\delta^{y}(x)=\delta_{\Lambda}(x-y)$ and $\theta_{\Lambda}^{y}(x)=\theta_{\Lambda}(x-y)$. Then $\theta_{\Lambda}^{y}(\infty)=1$. We let $\tilde{\theta}_{\Lambda}^{+}(y)$ be the solution of the wave equation having initial data $\left(\sqrt{\pi} \theta_{\Lambda}^{y}, \sqrt{\pi} \delta_{\Lambda}^{y}\right)$ and let $\tilde{\theta}_{\Lambda}^{-}(y)$ be the solution with initial data $\left(-\sqrt{\pi} \theta_{\Lambda}^{y}, \sqrt{\pi} \delta_{\Lambda}^{y}\right)$. By proposition (4.1) (i) the automorphisms $\gamma_{\theta_{A}^{+}(y)-\eta_{+}}$and $\gamma_{\theta_{\bar{A}}^{-}(y)-\eta_{-}}$are implemented in the Fock representation of $\Delta_{c}\left(M_{\alpha} \oplus M_{\xi}\right)$. In terms of the labelling introduced in the previous

1 We use their form of the Dirac matrices in the subsequent discussion 
section this means that they are implemented in $\mathscr{H}_{0,0}$ and hence in $\mathscr{H}_{m, n}$ for all $m, n \in Z$. By a slight abuse of notation we will write $W\left(\tilde{\theta}_{\Lambda}^{+}(y)-\tilde{n}_{\Lambda}^{+}\right)$and $W\left(\tilde{\theta}_{\Lambda}^{-}(y)-\right.$ $\tilde{\eta}_{-}$) for the operators which implement these automorphisms in

Regarding

$$
\mathscr{H}=\bigotimes_{m, n} \mathscr{H}_{m, n} \cdot
$$

$$
(t, x) \rightarrow W\left(\tilde{\theta}_{A} \pm(x \pm t)-\tilde{\eta}_{ \pm}\right) W\left(\tilde{\eta}_{ \pm}\right)
$$

as an operator valued distribution on $\mathscr{S}\left(\mathbb{R}^{2}\right)$, Dell-Antonio, Frischmann and Zwanziger show [29] that

$$
\lim _{\Lambda \rightarrow \infty} W\left(\tilde{\theta}_{\Lambda}^{ \pm}(x \pm t)-\tilde{\eta}_{ \pm}\right) W\left(\tilde{\eta}_{ \pm}\right)
$$

exists as an operator valued distribution (meaning of course that $\lim _{\Lambda \rightarrow \infty}$ is taken to be a distributional limit).

Now we consider the decomposition of $M_{2}$ into the direct sum of $M_{\alpha}$ and its orthogonal complement (with respect to the real inner product $\Gamma, \Gamma^{\prime} \rightarrow$ $\left.B\left(\Gamma, J_{F} \Gamma^{\prime}\right)\right)$, say $M_{\alpha}^{\perp}$. This decomposition gives a corresponding decomposition for $B$ into its (non-degenerate) restrictions to $M_{\alpha}$ and $M_{\alpha}^{\perp}$. As $M_{\alpha}$ and $M_{\alpha}^{\perp}$ are invariant under the complex structure $J_{F}$ we may define $\Delta_{c}\left(M_{\alpha}\right)$ and $\Delta_{c}\left(M_{\alpha}^{\perp}\right)$ as the CCR algebras over $M_{\alpha}$ and $M_{\alpha}^{\perp}$ and appeal to a theorem of Manuceau [16] to give

$$
\Delta_{c}\left(M_{2}\right) \triangleq \Delta\left(M_{\alpha}\right) \otimes \Delta_{c}\left(M_{\alpha}^{\perp}\right)
$$

with a corresponding decomposition

$$
\mathscr{H}\left(\pi_{F}\right) \equiv \mathscr{H}_{0,0} \triangleq \mathscr{H}_{0,0}^{\perp} \otimes \mathscr{H}_{0,0}^{2}
$$

where $\mathscr{H}_{0,0}^{i}, i=1,2$ carry the Fock representations of $\Delta_{c}\left(M_{\alpha}\right)$ and $\Delta_{c}\left(M_{\alpha}^{\perp}\right)$ respectively. This decomposition now carries over to $\mathscr{H}$ because

$$
\mathscr{H}_{m, n} \triangleq \mathscr{H}_{0,0}^{1} \otimes \mathscr{H}_{m, n}^{2}
$$

where $\mathscr{H}_{m, n}^{2}$ is defined in the obvious way (cf.discussion preceding (4.4)) so that

$$
\mathscr{H} \cong \mathscr{H}_{0,0}^{1} \otimes \underset{m, n}{\bigoplus} \mathscr{H}_{m, n}^{2}
$$

On the space $\mathscr{H}_{0,0}^{1}$ we have the Fock representation of a massive vector boson field which, by an abuse of notation, we write as an operator valued distribution $f \rightarrow \alpha_{\mu}\left(f^{\mu}\right)$ where $f: \mathbb{R}^{2} \rightarrow \mathbb{R}^{2}$ is smooth of compact support. Now it is a standard result in 2 dimensions that we can find a massive scalar boson field $\Sigma$ such that $g \alpha_{\mu}=-\varepsilon_{\mu \nu} \partial^{v} \Sigma$. Furthermore there is a Wightman field corresponding to the Wick ordered exponential : $\exp \pm i \sqrt{\pi} \Sigma:[20]$. We may therefore define

$$
\begin{aligned}
& \psi_{1}(t, x)=: \exp i \sqrt{\pi} \Sigma(t, x): \otimes \lim _{\Lambda \rightarrow \infty}: W\left(\tilde{\theta}_{\Lambda}^{+}(x \pm t)-\tilde{\eta}_{+}\right): \\
& \psi_{2}(t, x)=: \exp -i \sqrt{\pi} \Sigma(t, x): \otimes \lim _{\Lambda \rightarrow \infty}: W\left(\tilde{\theta}_{\Lambda}^{-}(x-t)-\tilde{\eta}_{-}\right):
\end{aligned}
$$

where the Wick ordering in the second factor means insert $W\left(\tilde{\eta}_{ \pm}\right)$between the 
annihilation and creation part of $W\left(\tilde{\theta}_{\Lambda}^{ \pm}(x \pm t)-\tilde{\eta}_{ \pm}\right)$(it is actually this Wick ordered limit which is defined in [30]).

These expressions for $\psi_{1}$ and $\psi_{2}$ were suggested by those of Lowenstein and Swieca [18] (where we have set their parameter $\alpha=\sqrt{\pi}$ ). Notice that the solution of Sec. III of [18] and the charge sectors defined in that paper involve an operator gauge condition. This operator condition does not make sense in our formulation, however an equivalent procedure is to regard $\mathscr{H}_{m, n}$ as the GNS Hilbert space corresponding to the pure state

$$
\sigma_{m, n}: \delta_{\Gamma_{\alpha}+\Gamma_{\xi}} \rightarrow \exp \left[i B_{0}\left(m \tilde{\eta}_{+}+n \tilde{\eta}_{-}, \Gamma_{\xi}\right)-\frac{1}{4} B\left(\Gamma_{\alpha,} J_{F}\left(\Gamma_{\alpha}\right)\right)\right]
$$

of $\Delta_{c}\left(M_{\alpha} \oplus M_{\xi}\right)$. These states then define charge sectors as before. However, the Fermion fields defined in [18] after the imposition of the operator gauge condition do not transform correctly under local guage transformations whereas those defined by (4.5) do.

We conclude with a number of remarks.

1. If we identify our field $A_{\mu}$ and the current $J_{\mu}$ defined in the previous section with the interacting electromagnetic potential and current respectively of [18] then $\partial_{\mu} \xi$ corresponds to the "longitudinal part of the current" (Eq. (2.13) of [18]) which is precisely what is set equal to zero in the operator gauge condition $((2.18)$ of [18]).

2. The original two Lagrangians for the Schwinger model imply that the field in $\Delta_{c}\left(M_{2}\right)$ is the analogue of $\partial^{\mu} A_{\mu}$ in $\Delta_{c}\left(M_{1}\right)$. With this identification we note that setting $J_{\mu}=g \alpha_{\mu}+\frac{1}{g} \partial_{\mu} \xi$ and $e A_{\mu}=e \alpha_{\mu}+\frac{\pi}{e} \partial_{\mu} \eta$, Maxwell's equations hold in the form

$$
\partial^{\mu} F_{\mu v}=-e J_{v}+\partial_{v} \xi
$$

which in (QED) $)_{4}$ would correspond to

$$
\partial^{\mu} F_{\mu \nu}=-e j_{v}+\partial_{v}\left(\partial^{\mu} A_{\mu}\right)
$$

compatible with what we would expect, in our approach, in (QED) ${ }_{4}$ [5].

3. The state (4.6) reduces on $\Delta_{c}\left(M_{\alpha}\right)$ to the Fock state. It is this fact which leads to the observation that the only observable of the Schwinger model is a massive boson field [18].

4. We return now to the discussion in the initial part of Sect. 3.4. In our version of the Lowenstein-Swieca sectors (4.6) we would be led to form the representation

$$
\sigma=\bigoplus_{m, n} \sigma_{m, n}
$$

and define charge shift operators in the obvious way. One would also define fermion fields on the resulting space (these would be those appearing in Sect. III of [18]) and moreover $\sigma \uparrow \Delta_{c}\left(M_{\alpha}\right)$ is then just a direct sum of Fock representations of a massive boson field and so no difficulty arises with positivity of the energy. As we remarked above (4.6) can be thought of loosely speaking, as imposing the operator condition $\xi \equiv 0$, effectively eliminating the local gauge transformations 
of the Fermions from the theory. Thus on the one hand if we want a faithful representation of the local gauge group and hence fermion operators transforming conventionally under the resultant gauge automorphisms we are led to (4.4) while on the other hand if we want positivity of the energy then (4.7) is suggested. So it does not appear to be possible to have both local gauge automorphisms and positivity of the generator of time translations in the Schwinger model.

We suspect that this difficulty is likely to persist in any definite metric approach to $(\mathrm{QED})_{4}$ which attempts to quantise the full 4 component vector potential. As we remarked previously the negative energies would cause no difficulties provided they were not dynamically coupled to the observable states.

Clearly an analysis of a more realistic model is required before firm conclusions can be drawn.

\section{References}

1. Carey, A. L., Gaffney, J. M., Hurst, C. A. : J. Math. Phys. (N.Y.) 18, 629-640 (1977)

2. Carey, A. L., Hurst, C. A. : J. Math. Phys. (N.Y.) 18, 1553-1561 (1977)

3. Carey, A. L., Gaffney, J. M., Hurst, C. A. : Rep. Math. Phys. 13, 419-430 (1978)

4. Carey, A. L., Hurst, C. A. : Lett. Math. Phys. 2, 227-234 (1978)

5. Carey, A. L., Hurst, C. A. : J. Math. Phys. (N.Y.) 20, 810-818 (1979)

6. Carey, A. L., Hurst, C. A., Keck, B. W. : Hadronic J. 1, 1144-1170 (1978)

7. Fermi, E. : Atti Accad. Lincei 9, 881 (1929); 12, 431 (1930); Rev. Mod. Phys. 4, 125 (1932)

8. Schwinger, J.: Phys. Rev. 125, 397 (1962)

9. Ito, K. R. : Prog. Theor. Phys. 53. 817 (1975) ; Construction of (QED) 2 . RIMS preprint (1977)

10. Lundberg, L.-E. : Commun. Math. Phys. 50, 103-112 (1976); Observable algebra approach to the Thirring-Schwinger model Preprint, Copenhagen (1976)

11. Fröhlich, J., Seiler, R. : Helv. Phys. Acta 49, 889-924 (1976) ; Nakanishi, N : Prog. Theor. Phys. 54, 840-841 (1975)

12. Doplicher, S., Haag, R., Roberts, J. E. : Commun. Math. Phys. 13, 1-23(1969); 15, 173-200 (1969)

13. Strocchi, F. : Phys. Rev. 162, 1429 (1967); D2, 2334 (1970)

14. Strocchi, F., Wightman, A. S. : J. Math. Phys. (N.Y.) 15, 2198 (1974)

15. Fröhlich, J. : Commun. Math. Phys. 66, 223-265 (1979)

16. Manuceau, J. : Ann. Inst. Henri Poincaré 8, 139-161 (1968)

17. Segal, I. E. : Mathematical Problems of Relativistic Physics. Am. Math. Soc. Providence 1963

18. Lowenstein, J. H., Swieca, Y. A. : Ann. Phys. 68, 172 (1971)

19. Seiler, R. Uhlenbrock, D. A. : Ann. Phys. 105, 81-110 (1977)

Leutwyler, H. : Helv. Phys. Acta 38, 431-447 (1965).

Klaiber, B. : In: Boulder lectures in theoretical physics, Vol. X-A, 1 New York: Gordon \& Breach 1967

20. Wightman, A.S. : In: Cargeselectures in theoretical physics, Vol. 1964. New York: Gorden \& Breach 1967

21. Kronig, R. de L. : Physica 2, 968-980 (1935)

Uhlenbrock, D. A. : Commun. Math. Phys. 4, 64-76 (1967)

22. Weingarten, D., Challifour, J. : Ann. Phys. (to appear)

23. Streater, R. F., Wilde, I. : Nucl. Phys. B24, $561-575$ (1970)

24. Emch, G. G. : Algebraic methods in statistical mechanics and quantum field theory. New York: Wiley-Interscience 1972

25. Strocchi, F.: Commun. Math. Phys. 56, 79-86 (1977)

26. Broadbridge, P. : Physica (to appear)

27. Morchio, G., Storcchi, F.: Infrared singularities, vacuum structure and pure phases in local quantum field theory. Preprint, Pisa 1979

28. Streater, R. F. : In: Physical Reality and Mathematical Description, Enz, C. P., Mehra, J. (eds.). Boston: Reidel 1974 
29. Dell-Antonio, G. F., Frischmann, Y., Zwanziger, D. : Phys. Rev. D6, 988 (1972)

30. Roberts, J. E. : Commun. Math. Phys. 51, 107-119 (1976)

31. Roberts, J. E. : Mathematical Problems in Theoretical Physics. In : Lecture Notes in Physics, Vol. 80. Berlin, Heidelberg, New York: Springer 1978

32. Private communication

Communicated by K. Osterwalder

Received December 11, 1979; in revised form July 1, 1980 
